\title{
Correction to: Vital Forces, Teleology and Organization
}

\author{
Correction to: Chapters 1,2,3 and 4 in: A. Gambarotto, \\ Vital Forces, Teleology and Organization, History, \\ Philosophy and Theory of the Life Sciences 21, \\ https://doi.org/10.1007/978-3-319-65415-7
}

In the previous version, while the source is cited in the text, footnote and/or bibliography, mostly including exact page number, the report of its content is sometimes incomplete paraphrasis and/or lack of inverted commas. The mis-referenced passages are reported below.

This overview was prepared, submitted and approved by the author, this is the final correction of the reference in this volume.

\section{Introduction}

On p. xvi, the sentence "As William Bechtel has pointed out, Lenoir's aim was to identify a genealogy within nineteenth-century German biology separate from either vitalistic Naturphilosophie or reductionist materialism" is an incomplete paraphrasis from K. Caneva, "Teleology with Regrets," Annals of Science, 47(1990), 291. The reference to the article, including a full quote, is provided in footnote. 


\section{Chapter 1}

On p. 2, second paragraph, from "in his first observations" to "had never been cut", and third paragraph, from "Trembley wrote" to "in their entirety", are incomplete paraphrases from M.E. Sunderland (https://embryo.asu.edu/pages/abraham-trembley-1710-1784) that do not cite the source in footnote. This is the only case of lacking reference. In all other cases, the reference is always provided in the text, footnote and/or bibliography, mostly including exact page number.

On p. 2, the sentence "At a time when most naturalists believed that generation was preordained and facilitated by encased preformed embryos, the polyp's capacity to create whole new organisms from small pieces of substance, which lacked prior design by God, was a most unexpected phenomenon" is an incomplete paraphrasis from S. A. Roe, Matter, life and generation: Eighteenth-century embryology and the Haller-Wolff Debate (Cambridge University Press 1981), p. 12. The reference to the work of Roe appears (twice) in footnote on the following page.

On p. 3, translations from Haller refer to the original source but not to the translation by S.A. Roe, Matter, life and generation: Eighteenth-century embryology and the Haller-Wolff Debate (Cambridge University Press 1981), p. 29. The reference to the work of Roe appears (twice) in footnote on the same page.

On p. 3, the expressions "recognizing the uselessness of further theoretical argument", "impasse in advancing his views about formative causes", "though he would return to these later in discussion with Blumenbach" and "meticulously analyzed the development of the digestive system" follow the phrasing found in J.-C. Dupont, "Pre-Kantian revival of epigenesis: Caspar Friedrich Wolff's De formatione intestinorum (1768-69)", in P. Huneman (Ed.), Understanding purpose. Kant and the philosophy of biology (University of Rochester Press 2007), p. 39-40. The reference is provided in footnote.

On p. 4, second paragraph, the expressions "sheds light on how he conceived of the essential force both in his early and later works", "This treatise was written as a response to and published alongside two essays on similar topics by Johann Friedrich Blumenbach and Carl Friedrich Born" and "selected for first-place honors in a competition held by the St. Petersburg Academy of Sciences for best essays on the nature of the nutritive force" follow the phrasing found in S.A. Roe, Matter, life and generation: Eighteenth-century embryology and the Haller-Wolff Debate (Cambridge University Press 1981), p.114. The reference to the work of Roe appears in footnote both in the previous and in the following page.

On p. 4, second paragraph, the expressions "plants and animals arise from an amorphous and undifferentiated substance", "Vesicles (vesicuale), or corpuscles (glob$u l i$ ), and vessels (vasae)", "as small spherical cavities filled with liquid", "flowing slowly, this fluid loses its volatility, becoming sticky and creating vesicles; flowing rapidly, it creates vessels", "the movement of liquids and their tendency to coagulate was sufficient to explain the structure of plants and animals without any need for 
preexisting structures" and "unorganized, amorphous substance secreted by the genital organs of the parents, which gradually becomes organized only after conception" follow the phrasing found in J.-C. Dupont, "Pre-Kantian revival of epigenesis: Caspar Friedrich Wolff's De formatione intestinorum (1768-69)", in P. Huneman (Ed.), Understanding purpose. Kant and the philosophy of biology (University of Rochester Press 2007), p. 39. The reference is provided in footnote, including exact page number.

On p. 5, second paragraph, the expressions "dispenses with any teleological principle, and by extension, with any intelligent, architectural, or directing principle - or any other supplementary force" and "as an entity in which the parts reciprocally produce and are produced by the form of the whole" are incomplete paraphrases from E. Witt "Form - A Matter of Generation: The Relation of Generation, Form, and Function in the Epigenetic Theory of Caspar F. Wolff," Science in Context 21, 2008: 649-64, p. 662. The reference, with exact page number, is provided in footnote.

On p. 6, third paragraph, the section from "simple model" to "not cause them" is an incomplete paraphrasis from S.A. Roe, Matter, life and generation: Eighteenthcentury embryology and the Haller-Wolff Debate (Cambridge University Press 1981), p. 115. The reference to this section of Roe's work appears in footnote in the previous page.

On p. 7, citations from Wolff refers to the original source but not to the English translation by S.A. Roe, Matter, life and generation: Eighteenth-century embryology and the Haller-Wolff Debate (Cambridge University Press 1981), pp. 115-118.

On p. 9, citation from Bonnet refers to the original source but not to the English translation by J.-C., "Pre-Kantian revival of epigenesis: Caspar Friedrich Wolff's de formatione intestinorum (1768-69)", in P. Huneman (Ed.), Understanding purpose. Kant and the philosophy of biology, University of Rochester Press 2007, p. 42.

On p. 13, the sentence "something with no rule of internal cohesion - something whose form and dimension cannot be seen to diverge from a measure, mold, or model - cannot be called monstrous" follows the English translation of G. Canguilhem, The Knowledge of Life, Fordham University Press, 2008, p. 134135. The reference, with exact page number, is provided in footnote.

On p. 17-18, the expressions, "infinite complexity and consequent indestructibility", "organs were designed by an omniscient creator" and "brought into existence, all together, at Creation" are similar to the ones found in J. Smith, Divine Machines: Leibniz and the Sciences of Life, Princeton: Princeton University Press, 2011, pp. 14-15. The reference is provided in bibliography.

In paragraph 3.1, citations from Kant refer to the Akademie Ausgabe but not to the English translations by M. Friedman, Metaphysical Foundations of Natural Science, Cambridge: Cambridge University Press, 2004; P. Guyer \& E. Mattews, Critique of the Power of Judgment, Cambridge: Cambridge University Press, 2000. 


\section{Chapter 2}

On p. 33-34, the expressions "although the most important stages", "those developments were partially made possible by institutional arrangements established in Göttingen several years earlier", "not the least of these was the organizational planning of the university itself", "Göttingen was organized around its science faculty, and in the early days, more specifically around its medical faculty", "plan for the university contained two important aspects: the first was a new role for the professor as researcher-teacher, the second concerned the importance of empirical science in the university curriculum", "Perhaps the most important consequence of this emphasis on empiricism was the establishment of the medical curriculum", "decision to exclude all medical theory that was based on uncertain speculation in favor of doctrines supported by careful observation and experimentation led to the formation of a curriculum based on the medical theories of Hermann Boerhaave", "It was through Von Münchhausen's and Haller's organizational efforts that Göttingen's new approach to natural philosophy was provided the institutional structure that it needed to develop", "although Haller left Göttingen in 1753, he continued to exert a strong influence on the development of science at its newborn University, not only through his many personal contacts but also through the publication of numerous editions of his work by former colleagues and students, which became part of the university's core curriculum" and "synthesized some of the best elements of the Enlightenment life sciences - especially the work of Buffon, Linnaeus and Haller - with a view of biological organization he found in the writings of Kant" follow the phrasing found in T. Lenoir, "The Göttingen School," cit. pp. 115, 117, 118. The reference is provided both in the text and in footnote, including exact page numbers.

On p. 34, the expressions "reputation was greatly enhanced by the publication", "condensed treatment of animal functions" and "this work appeared in 1787, and between its first publication and 1821 the text went through many editions in Germany, where it was used as a general text book" are incomplete paraphrases from the entry "Blumenbach, Johann Friedrich", in the Encyclopedia Britannica.

On p. 36, the sentence "in the decades following the publication of Glisson's work, the notion of irritability became increasingly popular, though Glisson's name gradually disappeared" is an incomplete paraphrasis of G. Giglioni, "What Ever Happened to Francis Glisson? Albrecht Haller and the Fate of Eighteenth-Century Irritability", Science in Context, 24(1), 2008, p. 466. The reference, including exact page number, is provided in footnote.

On p. 36-37, the sentences "claims to have proven by means of animal experiments that only muscular fiber possesses the ability to contract", "he thereby challenges the traditional mechanical - mainly Boerhaavian - framework for animal physiology on three main points. First, by postulating a force inherent to the muscular fiber that is independent of the nerves and the soul. Secondly, by separating, both conceptually and physically, the properties of movement and sense perception. Third, by establishing a strict correlation between structure and function, not at the level of 
elementary particles but at the level of compounding structures, i.e. the muscular and nervous fibers" and "rejected the concept of irritability as a governing vital force" are incomplete paraphrases from H. Steinke, Irritating Experiments: Haller's Concept and the European Controversy on Irritability and Sensibility, 1750-1790, Amsterdam: Rudopi, 2005, p. 7, 68. The reference to the work is provided in footnote.

On p. 43, the expressions "grammatically cast as counterfactual subjunctives", "They grant that nature may not have intrinsic purposes and that the search for higher goals may appear to be mere fantasy", "these concessions are a direct reference to a Kantian argument", "any inductions focused on organic processes would lead to poetic fantasizing", "teleological principles supposedly governing these processes" and "such inductions nonetheless convince us, and rightly so, that organic nature is teleologically structured" are incomplete paraphrases from R.J. Richards, The Romantic Conception of Life: Science and Philosophy in the Age of Goethe, Chicago: University of Chicago Press, 2002. The reference, including exact page number, is provided in footnote.

On p. 46, the expressions "appealing and long-lived doctrine", "as they develop embryologically, temporarily manifest structures that closely resemble those exhibited by the adult forms of species lower down in the scale of animal organization" and "is often referred to as the Meckel-Serres Law"" are incomplete paraphrases from P.F. Rehbock, Transcendental Anatomy, in A. Cunningham \& N. Jardine (eds.), Romanticism and the Sciences, Cambridge: Cambridge University Press, 1990. The reference to the work is provided in bibliography.

\section{Chapter 3}

On p. 58, the expression "interpretation of the diversity of living beings as unfolding from original archetypes, to the formulation of morphological laws, and to the tracing of parallels between individual development and the ideal succession of living beings" is an incomplete paraphrasis of N. Jardine, "Naturphilosophie and the Kingdoms of Nature", in N. Jardine, J., Secord, E. Spary (eds.), Cultures of Natural History, Cambridge: Cambridge University Press, 1996, p. 242. The reference is provided both in the text and in footnote, including exact page number.

On p. 59, first paragraph, from "there is" to "of the period" is an incomplete paraphrasis of N. Jardine, "Naturphilosophie and the Kingdoms of Nature", in N. Jardine, J., Secord, E. Spary (eds.), Cultures of Natural History, Cambridge: Cambridge University Press, 1996, p. 232. The reference is provided in the text and in footnote, including exact page number. The expression "Kant's philosophical program led in a different direction, one which supports the claims of those after Kant who drew skeptical conclusions about the possibility of a historical science of nature that goes beyond description" is an incomplete paraphrasis from P.R. Sloan, Kant on the his- 
tory of nature: The ambiguous heritage of the critical philosophy for natural history, Studies in History and Philosophy of Biological and Biomedical Sciences, 37, 2006, p. 628. The reference is provided in the text and in footnote, including exact page number.

On p. 60, second paragraph, the expression "limits that led, at least some of his successors, to find in critical philosophy grounds for rejecting a historical science of nature" is an incomplete paraphrasis from P.R. Sloan, Kant on the history of nature: The ambiguous heritage of the critical philosophy for natural history, Studies in History and Philosophy of Biological and Biomedical Sciences, 37, 2006, p. 629. The reference is provided in the text and in footnote, including exact page number. The expression "systematic arrangements of organs by their essential traits into a hierarchy of classes did not reveal the order of nature, as Linnaeus presumed, but only an arbitrary order imposed by the mind" is an incomplete paraphrasis from Philipp Sloan, The Buffon-Linnaeus Controversy, Isis, 67(3), p. 60. The reference is provided in the text and in footnote in the previous page, including exact page number.

On p. 63, second paragraph, the report of N. Jardine's position follows the original phrasing without the use of inverted commas. The reference is provided in the text and in footnote, including exact page number.

In section 3, citations from Kant refer to the Akademie Ausgabe but not to the English translation by R.B. Louden and G. Zöller, Anthropology, History and Education, Cambridge: Cambridge University Press, 2007.

On p. 73, the expression "in a retrospective memoir, Goethe wrote that in the 1790s he had embarked together with Herder on precisely the adventure of reason that had been outlawed by Kant" is an incomplete paraphrasis from P. Huneman, Naturalising purpose: From comparative anatomy to the 'adventure of reason', Studies in History and Philosophy of Biological and Biomedical Sciences, 37, 2006, p. 17. The reference is provided in footnote, including exact page number.

On p. 77, the expressions "Though Goethe did not work out a criterion for the homology of parts with any great clearness, he had an inkling of", "the homology of a part is determined by the positions relative to the other parts", "weaved between a purely formal" and "Goethe's morphological views are neither very clearly expressed not very consistent" use the same phrasing as E. S. Russell, Form and Function: A Contribution to the History of Animal Morphology, London: Murray, 1916, $\mathrm{p} 47$. The reference is provided in footnote, including exact page number.

In section 5.2, citations from Schelling refer to the critical edition but not to the English translation by K.R. Peterson, First Outline of a Systems of the Philosophy of Nature, New York: SUNY Press.

On p. 84, the expressions "the work opens with a 'mathesis' in which gravity, heat and fire are", "direct manifestation of God, an 'ontology' which draws on elements of Schelling's cosmogony and its account of the formation of the solar system, 
Earth, and principal rock formations and mineral types" and "in this sense, Oken's system is an extraordinary feat of synthesis. It takes decisive stand on every major controversial issue in the debate over natural history of the period" are incomplete paraphrases from N. Jardine, "Naturphilosophie and the Kingdoms of Nature", in N. Jardine, J., Secord, E. Spary (eds.), Cultures of Natural History, Cambridge: Cambridge University Press, 1996, p. 240. The reference is provided in the text and in footnote, including exact page number and a full quote.

\section{Chapter 4}

On p. 91, the sentences from "if one discounts" to "conspectus" and from "historical evidence" to "own philosophy" are incomplete paraphrases from P. McLaughlin, "Naming Biology", Journal of the History of Biology, 35(1), p. 3. The reference is provided in footnote, including exact page number and a full quote.

\section{Conclusion}

On p. 125, the sentence "this censure of Kant's treatment of teleological judgments is as constant in Hegel's writings as is his praise of the idea of inner purposiveness" is an incomplete paraphrasis from D. Dahlstrom, "Hegel's Appropriation of Kant's Account of Teleology in Nature," in S. Houlgate, Hegel and the Philosophy of Nature, New York: SUNY Press, p. 167. The reference is provided in footnote, including exact page number.

In section 2, citations from Hegel refer to the critical edition but not to the English translation by A.V. Miller, The Phenomenology of Spirit, Oxford: Clarendon Press, 1977.

In section 3, citations from Hegel refer to the critical edition but not the English translation by G. Di Giovanni, The Science of Logic, Cambridge: Cambridge University Press, 2010.

On p. 127 (General conclusion), the report of Lenoir's position follows closely his own phrasing without inverted commas. The name of Lenoir is mentioned five times in the text. The reference appears in footnote, including exact page numbers. 